\title{
Clinical-judicial syndrome: how a doctor becomes a patient through general indifference
}

\author{
Eugenio Santoro
}

Received: 21 May 2014/ Accepted: 8 August 2014/Published online: 24 August 2014

(C) Springer-Verlag Italia 2014

In recent years, disputes in the eternal and historical dialectic between doctors and patients, and between medicine and society, have increased. There has been a shift in our individual and social expectations from requesting treatment to demanding recovery, and searching for whoever is to blame if we do not obtain it.

With the legitimate demise of charismatic medicine and the growth of technological and participatory medicine, doctors are losing their central position in the healthcare process to protocols, the Internet and the mass media. There is, in short, an improvised and chaotic culture, often propagated by unqualified opinion-makers with little or no scientific background.

This has led to some mistaken convictions.

The first is that medicine is a science comparable to others, namely a mathematical and deductive process that can provide precise responses even to vague requests! however, even at the start of the twenty first century, medicine is still an exceedingly inductive and intuitive procedure that cannot easily be reduced to guidelines, no matter how broad.

The second is that, in adverse events such as complications, limited recovery, sequelae, disabilities, chronicity and death, it is not the disease that determines the rules and runs the show, however tragic, but the negligent or incompetent doctor, who, as such, is responsible for misfortunes and is the adversary of the patient.

This is the reason for the enormous increase in legal and judicial disputes and in civil and criminal actions against doctors, for which the responsibility is shared by the media,

E. Santoro $(\bowtie)$

Superior National Health Council and San Camillo Forlanini Hospital Foundation, Rome, Italy

e-mail: eugeniosantoro@tiscali.it the judges and their technical advisers, and the legislators. These are united in their hunt for the plague spreader, but have failed to provide a better definition of professional negligence or to establish a proper framework for vexatious litigation and the civil responsibility of judges, not only in regard to the outcome of the trial, but also to the interminable length of the proceedings.

Current opinion views medical negligence in relation to its insurance implications and to its social effects in terms of defensive medicine. No consideration, however, is given to the individual doctor facing charges and trial, to the man or woman, father or mother, worker, nurse, technician or health operative, in other words, to the human being. Yet they are seldom found guilty and almost always exonerated, after a procedure that is personally damaging, painful and costly, as well as a cause of emotional, personal and physical suffering to their families.

Current publications and conferences calculate the costs to patients and society in terms of insurance premiums, reimbursements and defensive medicine, but ignore the cost borne by those who are accused, who also end up as patients, in need of care, medication, check ups and rest, with absences from work, poor performance, and reduced productivity and earnings. Thus, a psycho-physical clinical syndrome develops, which can become chronic, irreversible and difficult to treat. Even when acquitted in court, no account is taken of the moral, economic and psychophysical harm suffered by the accused and their families or of the subsequent damage to the healthcare institutions for which they work.

Clinical-judicial syndrome was first described and defined in 1995 by Elias Hurtado-Hoyo and others, writing on behalf of the Argentine Medical Association. It is a series of symptoms that affect the health of an individual, in this case a doctor, subjected to legal proceedings, from 
their inception (filing of the claim, summons and notice of investigation), during the subsequent stages (resolution, mediation, committal for trial, hearings, rulings, appeals, etc.) and after their conclusion. It includes all the physical, psychological and behavioural alterations that can be caused in the doctor as a result of the legal events in which he or she is involved.

Certain predisposing factors can be present, including psycho-physical frailty, overworking, working in unsuitable conditions or with inadequate equipment, working in a critical field, a poor relationship with patients, the assumption of responsibilities greater than the level of academic and scientific training, and poor economic compensation. It has also been shown that the level of susceptibility in medicine and surgery, as in all other occupations, is related to individual lifestyle habits (diet, smoking, alcohol, drugs, physical activity, sleep, etc.), psychological adaptation to the environment and work (stress), and social integration.

The legal action is the triggering factor. Two distinct pathogenic aspects are associated with the filing of the claim, both of which lead to stress. The first is the sudden and unexpected arrival at the doctor's home of notification of the claim for liability or the initiation of legal proceedings. The second is the categorical and aggressive written language normally used by the injured parties, or rather, their lawyers. This can contain unsubstantiated allegations, negative details of no clinical relevance, statements by the patients or their relatives that are at times offensive or irrational, or else harmful to the doctor's professional reputation, clinical evaluations with no scientific basis and disproportionate financial requests. All of this tends to aggravate the doctor's situation. These charges, even if proven unfounded, can have a very negative impact on the doctor's state of mind, mental and physical health, family relationships and personal and professional behaviour.

Aggravating factors should also be borne in mind, including: other patients and their contentious relatives, negative propaganda in the workplace, spitefulness and gossip by colleagues and collaborators, and the notoriety given to the event and the charges by the mass media.

The committal for trial is the most serious event, placing the accused doctor in a state of anxiety and uncertainty that can trigger clinical-judicial syndrome, thus affecting his or her life, first as an individual and then as a professional.

Some accused physicians manage to maintain a sufficient state of equilibrium, without any externally visible or admitted symptoms, but most report acute or chronic stress, with complex clinical symptoms combined, in varying degrees, with physical, psychological and behavioural changes. The physical effects can include: bronchial asthma, digestive haemorrhages, myocardial infarction, arterial hypertension, headache, cerebral vascular accidents, diarrhoea, skin diseases, immunodepression and fatigue. Psychological consequences can involve: anxiety, depression, paranoia, emotional instability, irritability, sexual dysfunction, insomnia and other effects. Behavioural changes can include: mental block, obsessive ideas, moral frustration, depersonalisation, loss of self-esteem, isolation, disruption of behaviour and family and social relationships with relatives, patients and colleagues at the health institution where they work, a medically defensive professional approach and even excessive use of drugs, cigarettes, alcohol, etc.

The conclusion reached by all the authors who studied the subject is that no doctor is ever the same doctor or person after facing judicial proceedings. Therefore, every trial for suspected or proven professional liability always has a guaranteed loser, namely the physician, as a professional and individual, even if the final judgement is in his of her favour.

Society, represented by medical, political and judicial institutions, together with the relevant professional, trade union, citizens' and patients' associations and mass media, does not seem interested in the personal fate of the individual doctor, whether as victim or perpetrator of the judiciary event, despite the evident fact that no intentionality is ever attributed to his or her actions.

Great attention is paid to the insurance and monetary aspects of the event, as well as to the consequences for healthcare in terms of defensive medicine, which is more expensive and less effective. No one, however, takes any responsibility for the personal, health and social problems of the involuntary protagonist or for his or her mental and physical health.

Yet the infirmity caused to the individual doctor by the judicial proceedings also has a direct and indirect cost. The direct cost involves the analyses, examinations, treatments, medicines, hospitalisations and absences from work that are the inevitable consequences of clinical-judicial syndrome as described above. The indirect cost, which goes far beyond defensive medicine, is the social cost of the individual's marginalisation or self-withdrawal and the cost of employing a professional who is fearful, listless, under-performing, present at work less continuously and with a diminished capacity for decision-making, diagnosis and therapy.

Healthcare disputes are being allowed to proliferate, with daily newspapers and periodicals free to publish invitations to bring charges against doctors and hospitals, and state television allowed to act as a megaphone for prospectors in the new goldmine of alleged medical malpractice, instead of protecting the professional heritage of 
the National Health Service. ANIA states that in recent years there have been at least 40,000 complaints annually, involving no less than 20,000 physicians. At this rate, in approximately 10 years the number of doctors that have been subjected to legal proceedings will be around 200,000 , i.e. half the total number of medical practitioners in Italy. The Italian National Health Service risks being seriously undermined and, above all, clinical-judicial syndrome will end up becoming a genuine epidemic!

Rome, 10 January 2014.

Conflict of interest None. 